\title{
TRABAJO INFANTIL, JUSTICIA DISTRIBUTIVA Y ECONOMÍA DEL BIENESTAR
}

EDUARDO CALDERÓN CUEVAS

Universidad Autónoma de Barcelona

\author{
PALABRAS CLAVE ADICIONALES ADDITIONAL KEYWORDS \\ Pobreza, Utilitarismo, Igualdad de Oportunidades, Poverty, Utilitarism, Equality of Opportunities, \\ Capacidades y Funcionamientos. \\ Functioning and Capabilities.
}

RESUMEN. El objetivo de este artículo es estudiar desde los postulados de la economía del bienestar o normativa cómo se justifica la participación del Estado para prohibir, regular o reducir el trabajo infantil. Se señalarán sus limitaciones (como teoría de justicia distributiva y se explorarán criterios alternativos de distribución como el principio de diferencia de Rawls, la igualdad de oportunidades de Roemer y las capacidades de Sen), que podrían ser más acordes para valorar y justificar el tipo de políticas distributivas que se necesitan para resolver esta problemática social.

SUMMARY. The main purpose of this paper is to justify a governmental intervention to reduce Child Labour based on the principles of the Welfare Economics. The conclusion states that Welfare Economics has important limitations when it comes to justify a larger resource allocation in order to eliminate children and youth participation in labour market. According to this, this paper presents alternative criteria refering to distributive justice with special emphasis on Child Labour, such as Rawls' difference principle, Roemer's equality of oportunity principle and Amartya Sen's functioning-capabilities principle.

* Este trabajo sc enmarca dentro del proyccto Globalización y desigualdades en América Latina: formación, contenidos e impactos de las políticas educativas y de lucha contra la pobreza en Argentina, Brasil y Chile, financiado por cl Ministerio de Ciencia y Tecnología y el FEDER (proyectos I+D) con referencia SEC2002-02480. Agradezco los comentarios y sugerencias de José Antonio Noguera, José Adelantado, Xavier Bonal y Norma Alicia del Río sobre las versiones anteriores de este documento. Muy en especial a Silvia Tort por su apoyo para poder realizar la investigación.

E-mail: eduardo.calderon@uab.es

Revista Internacional de Sociología (RIS)

Tercera Época, $N^{\circ} 36$, Septiembre-Diciembre, 2003, pp. 33-57. 


\section{"El hecho de que una persona haya aprendido a vivir en medio de la adversidad y la pobreza, aprendiendo a sonreir valientemente ante ella, no debe anular su derecho a ser compensada" \\ Amartya Sen.}

\section{INTRODUCCIÓN}

En su esfuerzo por formalizar un modelo económico que refleje las causas y consecuencias del trabajo infantil, la economía positiva ha olvidado trazar líneas de unión con su vertiente normativa para lograr una construcción más sólida de cómo abordar este tema y responder a preguntas tales como: ¿se puede considerar justa a aquella sociedad que acepta y necesita desigualdades como el trabajo infantil? ¿Cómo se justifica la intervención del Estado para eliminar el trabajo infantil? ¿Cuál debe ser la naturaleza de esta intervención en materia distributiva?

La economía del bienestar estudia y aplica juicios valorativos frente a las políticas gubernamentales. Dado que de ella depende la función de valorar si una asignación o distribución de los recursos es socialmente justa, es importante investigar más a fondo la base de sus principios. Esto nos permitirá delimitar el tipo de políticas distributivas que entrarían dentro de su esquema, y dado que nuestro objeto de estudio es el trabajo infantil, poder reflexionar sobre cómo se justificaría una intervención en este campo y cuál sería su naturaleza. De esta forma contaríamos con las herramientas necesarias para criticar sus argumentos y defender políticas distributivas de mayor escala.

Antes de profundizar en la discusión es importante dejar muy claro qué se entiende por trabajo infantil (TI, en adelante). En la literatura se tiende a diferenciar entre el concepto de "niños económicamente activos" (NEA) que comprenden todas las actividades productivas realizadas por los niños incluyendo los trabajos no remunerados, ilegales y en el sector informal, frente al concepto de trabajo infantil. Para la Organización Internacional del Trabajo (OIT) el trabajo infantil se refiere, además de a los NEA, al trabajo que necesita ser eliminado según las convenciones sobre la edad mínima (Convenio no. 138, 1973) y sobre las peores formas de trabajo infantil (Convenio no. 182, 1999).

En este último convenio se acordó que, según el tipo de actividad, las peores formas de trabajo infantil se pueden clasificar en labores peligrosas, hazardous work, (trabajos cuyos efectos puedan causar a los niños daños físicos, psicológicos o morales, y también se estableció un límite de 43 horas a la semana que si se supera entran dentro de esta categoría) y la otra clasificación que se adoptó son los trabajos inaceptables, unconditional worst forms, como: tráfico de niños, trabajos forzados, niños involucrados en conflictos armados, pornografia y prostitución infantil, y niños que trabajen en actividades ilícitas, como la producción o distribución de droga. 
Tabla 1.

\begin{tabular}{|c|c|c|c|c|}
\hline \multirow{3}{*}{$\begin{array}{l}\text { Grupos } \\
\text { de Edad }\end{array}$} & \multicolumn{4}{|c|}{ Tipos de Trabajo Infantil } \\
\hline & \multicolumn{2}{|c|}{ Niños económicamente activos } & \multicolumn{2}{|c|}{ Peores formas de Trabajo Infantil } \\
\hline & $\begin{array}{c}\text { Trabajo ligero }^{1} \\
(<14 \text { horas } \\
\text { semanales })\end{array}$ & $\begin{array}{c}\text { Trabajo regular } \\
\text { ( }<43 \text { horas } \\
\text { semanales })\end{array}$ & $\begin{array}{c}\text { Trabajos } \\
\text { peligrosos }\end{array}$ & $\begin{array}{c}\text { Trabajos } \\
\text { inaceptables }\end{array}$ \\
\hline $5-11$ & & & & \\
\hline $12-14$ & & & & \\
\hline $15-17$ & & & & = \\
\hline
\end{tabular}

* Las áreas sombreadas son las que la OIT considera necesaria su eliminación.

Por lo tanto, el trabajo infantil según tramos de edad comprendería a los niños y niñas según la tabla 1.

La principal crítica a esta definición es que la OIT no considera el trabajo doméstico como trabajo infantil. Es importante incluir en nuestra discusión el trabajo que se realiza dentro del hogar, en donde la participación de las niñas es mucho más alta, ya que este tipo de actividad juega es papel determinante en sus decisiones de asistir a la escuela o bien cuidar del hogar y de los hermanos pequeños.

De lo anterior, se deriva una gran gama de tipo de actividades que llegan a realizar los niños, con lo cual se parte de que el trabajo infantil es un fenómeno complejo, con múltiples matices, y para lograr su eliminación es indispensable intervenir en las siguientes tres áreas: a) en protección; b) en el desarrollo de los niños y niñas²; y c) en el control de los efectos negativos que ocasiona (Anker, 2000).

a) En el área de protección las políticas han sido más de carácter legislativo (de prohibición y regulación), debido a que es común que los niños trabajadores estén más expuestos a sufrir abusos y explotación. Al respecto, se han producido importantes avances como la firma del Convenio $n^{\circ} 138$ en 1973 sobre la edad mínima laboral y el Convenio $\mathrm{n}^{\circ} 182$ firmada en 1999 sobre las peores formas de trabajo infantil, con el objeto de crear un consenso internacional en la definición y prohibición del trabajo infantil, facilitando también así su identificación y medición.

\footnotetext{
${ }^{1} \mathrm{La}$ OIT considera que hasta 14 horas semanales el trabajo ligero no tiene ningún efecto negativo sobre el rendimiento escolar del niño.

${ }^{2}$ En adelante se utilizará, por conveniencia, sólo el término de niños para referirnos a ambos sexos pero sin la intención de excluir a las niñas y menos aún en el tema de trabajo infantil, donde los análisis por género señalan condiciones y comportamientos totalmente distintos.
} 
b) Con relación al desarrollo de los niños, la principal preocupación es que el TI interfiere con su vida académica, con lo cual en el largo plazo esta vivencia puede determinar el tipo y calidad de vida a la que pueden aspirar. Lo anterior se debe matizar dependiendo del tipo de actividad que realicen los niños y el tiempo que le dediquen.

Además, el debate se enriquece al ampliar nuestra forma de entender el desarrollo de los niños si consideramos, como se da en muchas regiones y culturas, que las habilidades y conocimientos se pueden adquirir tanto en la educación formal (saber leer, escribir y operaciones aritméticas), como por la experiencia en el trabajo y en la vida (responsabilidad, disciplina, entre otras).

c) Sobre el control de los efectos negativos que ocasiona el TI, se pueden clasificar en dos áreas: a nivel micro y macro. Los efectos micro están relacionados con el estudio de la pobreza dentro del hogar. En muchas ocasiones el trabajo de los niños es necesario como estrategia de supervivencia del hogar, con lo cual es importante estudiar los impactos que puedan generar las políticas orientadas a prohibir el TI en el bienestar total del hogar. Dependiendo del tipo de actividad que realicen los niños se debe diferenciar entre políticas focalizadas, o bien políticas de carácter más universal que contemplen sus diversos determinantes ${ }^{3}$.

A nivel macro los efectos se observan en el mercado laboral, en el comercio internacional, en el crecimiento y en desarrollo económico. Con relación al mercado laboral, la idea común es creer que el trabajo infantil desplaza el trabajo de los adultos y, como consecuencia, se reduce el nivel salarial o se incrementa la tasa de desempleo, o ambas cosas ${ }^{4}$. Sin embargo, este análisis sólo es válido en las actividades donde el niño esté realmente integrado al mercado laboral mediante un trabajo asalariado, por tanto, no es aplicable a las actividades no remuneradas que se realizan en trabajos familiares o dentro del hogar y que en gran medida son llevadas a cabo por las niñas. Asimismo, un área de intervención clara y muy justificada desde la economía se centra en estudiar y controlar los efectos del TI sobre el crecimiento y desarrollo económico (Basu y Van, 1998). Buscan romper el círculo vicioso intergeneracional que genera el trabajo infantil: la pobreza de los padres limita la inversión en educación de sus hijos al enviarlos a trabajar, con lo cual esta generación a la larga tendrá menores oportunidades de obtener ingresos, incrementándose el riesgo de que ese individuo viva en la pobreza y se

\footnotetext{
${ }^{3}$ Los programas que se han desarrollado en México (Oportunidades, antes Progresa) y en Brasil (Bolsa-Familia) son un claro ejemplo de los esfuerzos que se han aplicado por integrar las politicas sociales con las económicas a fin de combatir desde sus múltiples causas los problemas como el trabajo infantil.

${ }^{4}$ Estos efectos pueden ser especialmente mayores en los países en desarrollo, debido a que sus sistemas productivos están basados más en emplear trabajadores poco cualificados facilitándose la sustitución entre trabajo de adultos y niños.
} 
perpetúe esta condición sobre una nueva generación, con los respectivos costes de productividad, crecimiento y desarrollo económico que esto implica.

Teniendo claras estas áreas de intervención, el objetivo de este trabajo es estudiar desde los postulados de la economía del bienestar o normativa cómo se justifica la participación del Estado para prohibir, regular o reducir el trabajo infantil. Se señalarán sus limitaciones en materia distributiva y sus incompatibilidades para integrar en su análisis esta problemática social sin violar intuiciones morales básicas.

El trabajo está organizado de la siguiente forma: en el segundo apartado se mencionan, desde la perspectiva económica, los principales determinantes del TI. En la tercera sección, se analiza cómo la economía positiva ha estudiado el TI y se presenta un cuadro con sus principales teorías: desde el modelo más simple de negociación dentro del hogar enunciado por Becker (1964), hasta los modelos dinámicos de generaciones solapadas como el de Basu y James Foster (1998) y el de López-Calva (2002). También en este apartado se discute sobre la respuesta más común de la economía normativa al problema del TI, dejando para el cuarto apartado el desarrollo de una crítica más profunda a su base normativa con reflexiones sobre el TI.

En el quinto apartado se exploran las principales contribuciones de los últimos años por parte de economistas normativos como John Roemer, Amartya Sen, y de filósofos políticos como Rawls, para utilizar criterios normativos alternativos a considerar, a la hora de evaluar el impacto de las políticas orientadas a la disminución o eliminación del trabajo infantil y finalmente se presentan algunas. La última sección se reserva para las conclusiones.

\section{DETERMINANTES DEL TRABAJO INFANTIL}

La discusión tanto política como académica sobre el trabajo infantil ha tomado mucha importancia en los últimos años a nivel internacional ${ }^{5}$. Desde la economía se ha generado un gran número de investigaciones sobre las causas y consecuencias del trabajo infantil (véase Basú, 1999). Entre sus principales contribuciones se encuentra el hecho de mostrar que el TI es un fenómeno más ligado al subdesarrollo y a la pobreza, es decir, que mediante el estudio de su evolución se

\footnotetext{
${ }^{5}$ La discusión tanto política como académica sobre el trabajo infantil entró de nuevo en la agenda internacional tras la polémica propuesta Child Labor Deterrence Act, también conocida como la "Harking Bill", que fue presentada en 1995 al Congreso de los Estados Unidos para prohibir las importaciones de todos los productos que en su proceso productivo utilizaban cualquier tipo de trabajo infantil. No se debe ignorar que el espíritu de esta propuesta era más bien de carácter proteccionista. frente a la idea de un posible dumping social de los países pobres.
} 
puede apoyar la idea de que el crecimiento y el desarrollo económico disminuye su incidencia ${ }^{6}$.

Partiendo de esta premisa, es importante delimitar cuáles son los principales determinantes del trabajo infantil. Con base en el trabajo de Grootaert y Kanbur (1995), en donde realizan una síntesis interesante de las investigaciones que se habían desarrollado en la materia, a continuación se presenta una agrupación de los determinantes por el lado de la oferta y la demanda de TI.

\section{Determinantes desde la oferta}

A) El tamaño del hogar y la asignación del tiempo. Las decisiones sobre la asignación del tiempo de los niños dependen de su productividad potencial y de la de sus padres, del grado de sustitución del trabajo entre ambos, pero, sobre todo, de la estructura y del tamaño del hogar. La relación esperada es que los hogares numerosos reducen la participación y el progreso educacional de los niños (especialmente si tienen hermanos menores), ya que los padres no tienen incentivos en invertir en el capital humano de sus hijos, o bien sus costos son muy altos (Lloyd, 1993). Dada la heterogeneidad de las actividades del trabajo infantil (en la calle, en las plantaciones, en las fábricas, en los mercados fijos y ambulantes, como recolectores de basura y muchas más), el efecto de estas variables puede ser distinto dependiendo de si el estudio se centra en las tareas a tiempo completo o parcial, en el trabajo de mercado o doméstico, o bien si se está analizando el ámbito rural o urbano, como bien lo señala en su estudio sobre Filipinas DeGraff (1993).

B) Características socio-económicas de los responsables del hogar. Los resultados más sólidos en los estudios empíricos que se han realizado en diferentes regiones muestran que el nivel de educación de los padres y su situación laboral son los factores que más pesan en la decisión de enviar a sus hijos a trabajar o a estudiar (Psacharopoulos, 1997; Gustafsson-Wright Pyne, 2002; Levison Deborah, 2001). El grado de segmentación del mercado laboral, las desigualdades salariales, y su nivel de flexibilización son algunos de los elementos que influyen determinantemente en el tipo de oportunidades que tienen los jefes del hogar, en su nivel de pobreza y, por ende, en el trabajo infantil como estrategia de supervivencia. Investigaciones muy interesantes que diferencian por género como la de Alaka Basu (1993) en algunos barrios de las afueras de Nueva Delhi, muestran cómo

\footnotetext{
${ }^{6}$ Estudios históricos que parten de la revolución industrial, como el de Cunningham (1990), muestran las tendencias de reducción en la tasa de participación de los niños en diferentes países después de haber registrado altos niveles a principios y mediados del siglo XIX como el caso de Inglaterra. Ello apoyaría la idea de que el crecimiento y desarrollo es necesario, más.no suficiente, para disminuir el trabajo infantil.
} 
las condiciones en las que se encuentran los responsable del hogar con relación al mercado laboral afectan sus decisiones sobre el uso del tiempo del niño. Señala que las madres, al participar en la fuerza laboral o al buscar mayores ingresos, sustituyen su trabajo doméstico por el de mercado y, dado que por lo general los salarios a los que pueden acceder no son suficientes para contratar a alguien que realice los trabajos del hogar, sacan de la escuela a sus hijas o las ponen a trabajar a tiempo parcial en el mejor de los casos ${ }^{7}$. La pregunta abierta en este contexto es: ¿qué nivel de ingresos es necesario garantizar a los padres (en especial a las madres) para que se evite el efecto substitución educación-trabajo doméstico?

C) Relación entre Educación y Trabajo Infantil. Estudios recientes sobre la relación entre educación y trabajo infantil parten de una concepción de actividades que no son mutuamente excluyentes, sino que se complementan entre sí. Algunas investigaciones como las de Grootaert (1998) para Cote d'Ivoire, de Psacharopoulos (1997) para Perú, de Gustafsson-Wright y Pyne (2002) para Brasil, y de Levinson, Moe y Knaul (2001) para México, revelan que el trabajo infantil a tiempo parcial no actúa en detrimento de la escolaridad del niño principalmente en las zonas rurales o en el trabajo informal de las ciudades, con lo cual cabría preguntarse si su trabajo es realmente lo que le permite estudiar y si sólo así puede acceder a mejores oportunidades de vida. Asimismo, otro punto que hay que destacar es el que aborda Bonnet (1993) en sus investigaciones sobre África acerca de las condiciones del sistema educativo. Concluyen que el sistema educativo también tiene la función de reproducir las desigualdades sociales y su capacidad de garantizar un puesto de trabajo es limitada generándose, así, una influencia positiva para la oferta de trabajo infantil. En este sentido, cuando los padres valoran la calidad y eficiencia del sistema educativo al que enviarían a sus hijos, algunas veces encuentran que la educación no es el camino para garantizarle un trabajo estable y bien retribuido. Estos tipos de decisiones son muy comunes en los grupos sociales de bajos ingresos debido a que sus oportunidades de empleo se ubican más en el sector informal en donde la inversión en Capital Humano pierde relevancia.

D) Riesgos que enfrenta el hogar. El principal riesgo es la inseguridad económica; investigaciones como la de Jacoby and Skoufias (1994) confirman que el trabajo infantil funciona como un seguro del hogar que utilizan ante situaciones inesperadas (pérdida del empleo de un miembro de la familia, de emergencias en salud, entre otras). Si se aplicaran políticas de prohibición, éstas fracasarían en su intento por mejorar las condiciones de vida de los niños si no van acompañadas

\footnotetext{
${ }^{7}$ A esta relación la han identificado como la " $U$ " invertida entre los salarios de las madres y el trabajo infantil.
} 
de mecanismos de seguridad contra fluctuaciones como la provisión de créditos a corto plazo sin requerir de algún colateral.

\section{Determinantes desde la demanda}

A) Las condiciones del Mercado Laboral y la demanda de trabajo infantil. Es evidente que el grado de segmentación del mercado laboral entre empleos formales e informales es un importante determinante en el trabajo infantil, ya sea indirectamente, determinando las condiciones de trabajo de los responsables del hogar como se explicó en el apartado anterior, o bien directamente, por la fijación del nivel salarial al que puede acceder un niño. En este sentido, la probabilidad de que los niños se vean incentivados a trabajar dependerá de su salario potencial, que a su vez estará determinado por el hecho de que el sector al que pueda acceder sea formal o informal. La tendencia de los mercados laborales en los países en desarrollo es hacia una alta participación del sector informal en los métodos de producción y de distribución, con lo cual existe un amplio espacio receptor de mano de obra infantil donde las actividades están poco reguladas y muy flexibilizadas (Levinson, Moe y Knaul, 2001). Asimismo, las estrategias empresariales para evadir legislaciones sociales y reducir sus costes se han enfocado en la subcontratación hacia organizaciones que operan en el sector informal o bien a nivel incluso de empresas familiares. Debido a estas fuerzas dirigidas hacia la in-formalización, se puede esperar que exista una mayor presión de la demanda de trabajo infantil.

B) El papel de la tecnología. En un sistema productivo dado, la influencia de la tecnología en el TI se da vía el grado de sustitución entre el trabajo de los adultos y el de los niños. Algunos de los cambios tecnológicos que se han presentado a lo largo del tiempo, han influido tanto positiva como negativamente en el trabajo infantil. Éste es un campo aún poco explorado por las investigaciones empíricas dada la dificultad de su análisis por la ambivalencia de los efectos del cambio tecnológico y debido a la ausencia de datos fiables. Ejemplos de lo anterior son: mediante la mecanización de la agricultura al introducir tractores o un sistema de riego avanzado, o bien tan sólo garantizando el servicio de electricidad en un hogar se ha reducido indirectamente la demanda de TI. Sin embargo, otro tipo de innovaciones tecnológicas como la miniaturización de los componentes electrónicos, pueden ocasionar incrementos en el TI debido a las estrategias de subcontratación de las empresas para que los niños trabajen desde su hogar ensamblando estos materiales. 


\section{ECONOMÍA POSITIVA Y NORMATIVA}

Habiendo identificado los determinantes del TI, es necesario abordar el tema de cómo la economía ha intentado formalizar el proceso causal y las consecuencias de este fenómeno, así como el tipo de juicios valorativos que pueden derivarse de sus postulados. Lo anterior nos permitirá delimitar el tipo de políticas que recomendaría instrumentar la economía para combatirlo y la forma en que justificaría la intervención del Estado para garantizar un particular tipo de justicia distributiva ${ }^{8}$.

\section{Estudios desde la Economía Positiva}

La mayoría de los modelos formales que la economía positiva ha desarrollado justifican la intervención del gobierno para eliminar o regular el trabajo infantil bajo argumentos de eficiencia y externalidades, principalmente la externalidad positiva de la educación.

Un claro ejemplo de este argumento se puede ver en el trabajo de Grootaert and Kanbur (1995), los cuales consideran que las tasas de retorno social de la educación pueden exceder a las tasas de rendimiento privadas; en este sentido, la intervención del gobierno para que los niños en vez de trabajar estén estudiando sería deseable.

El caso extremo de externalidad se encuentra en el modelo de Gupta (1997) pues considera que los padres y el agente que contrata al niño toman la decisión exclusivamente velando por sus propios intereses y utilizan al niño como un mero instrumento, con lo cual los efectos sociales son nulos.

En el cuadro 1 se pueden observar las principales áreas de estudio donde la economía positiva ha incursionado para desarrollar sus modelos teóricos sobre trabajo infantil ${ }^{9}$. Su interés se centra en comprender el proceso de decisiones dentro y fuera del hogar en un modelo unitario (Becker, 1964) o de negociación, explorando la heterogeneidad del mercado laboral y su relación con el trabajo infantil mediante modelos de equilibrio múltiple, y buscando derivar conclusiones sobre las consecuencias del trabajo infantil mediante los modelos dinámicos de generaciones solapadas, como el de López Calva (2002).

\footnotetext{
${ }^{8}$ Aún falta mucho camino por recorrer para llegar a un acuerdo estable y fijar unas alianzas sólidas entre la Economía Positiva, del Bienestar y la Filosofia Política, pero resulta destacable mencionar las aportaciones que han realizado las dos primeras al estudio de la justicia distributiva mediante el uso de herramientas matemáticas. Coincido con Sen y Doménech que más vale tener la valentía de equivocarse con precisión de vez en cuando, que acertar siempre sirviéndose de la confusión y la vaguedad conceptuales.

${ }^{9}$ Este esquema está basado en el artículo de Basu (1999) donde se detalla la propuesta de cada autor por si al lector le interesa profundizar en el tema y tener las referencias exactas.
} 
R I S

REVISTA INTERNACIONAL DE SOCIOLOGI

Cuadro 1.

Principales modelos teóricos sobre trabajo infantil.

\begin{tabular}{|l|l|l|}
\hline \multicolumn{3}{|c|}{ I. MODELOS DE NEGOCIACIÓN } \\
"Bargaining": Depende de quiénes son los involucrados en la negociación ${ }^{10}$ \\
I.
\end{tabular}

${ }^{10}$ Los modelos de negociación por su naturaleza excluirían en su análisis a los niños de la calle. 


\begin{tabular}{|c|c|c|}
\hline \multicolumn{3}{|c|}{$\begin{array}{l}\text { II. EQUILIBRIO MÚLTIPLE E INTERVENCIÓN DEL GOBIERNO: Buscan enriquecer el } \\
\text { análisis de los modelos anteriores considerando que el mercado laboral, donde los niños son traba- } \\
\text { jadores potenciales, es muy probable que presente más de un equilibrio dependiendo del salario al } \\
\text { que puede acceder un adulto, con lo que se abren distintos tipos de politicas gubernamentales. }\end{array}$} \\
\hline $\begin{array}{l}\text { A) Modelo con } \\
\text { altruismo }\end{array}$ & $\begin{array}{l}\text { Introducen la posibilidad de múltiples } \\
\text { equilibrios }\end{array}$ & \\
\hline & $\begin{array}{l}\text { - Esta basado en dos grandes supuestos: } \\
\text { Luxury axiom y el de Substitution Axiom. El } \\
\text { primero se refiere a que los padres no enviarían } \\
\text { a sus hijos a trabajar si sus ingresos fueran lo } \\
\text { suficientemente altos. El segundo axioma } \\
\text { contempla el grado de sustitución entre los } \\
\text { niños y adultos, es decir, que los niños pueden } \\
\text { hacer actividades similares a los adultos. }\end{array}$ & Basu and Van (1998) \\
\hline \multirow{2}{*}{$\begin{array}{l}\text { B) Relación de } \\
\text { Trabajo Infantil con } \\
\text { normas sociales }\end{array}$} & $\begin{array}{l}\text { La decisión de enviar al niño a trabajar se debe } \\
\text { en parte a normas sociales. }\end{array}$ & * \\
\hline & $\begin{array}{l}\text { - Dependiendo de la comunidad donde se } \\
\text { encuentre, que los hijos trabajen puede signifi- } \\
\text { car un costo de estigma social, o a la inversa, si } \\
\text { el número de niños que trabajan es alto en esa } \\
\text { comunidad. }\end{array}$ & Basu (1999) \\
\hline \multirow{3}{*}{$\begin{array}{l}\text { C) Relación con el } \\
\text { tipo de distribución } \\
\text { y el desempleo de } \\
\text { los adultos }\end{array}$} & $\begin{array}{l}\text { Campo abierto en el desarrollo de modelos } \\
\text { teóricos, sin embargo, existen algunas } \\
\text { estimaciones empiricas. }\end{array}$ & \\
\hline & - Sobre la distribución del ingreso & $\begin{array}{l}\text { Kenneth Swinnerton y } \\
\text { Carol Ann Rogers } \\
\text { (en prensa) }\end{array}$ \\
\hline & $\begin{array}{l}\text { - Sobre la relación entre el desempleo de los } \\
\text { padres y el trabajo infantil }\end{array}$ & $\begin{array}{l}\text { Michael Bonnet } \\
\text { (1993); Cahndrasekhar } \\
\text { (1997) }\end{array}$ \\
\hline \multirow[t]{5}{*}{ D) Dinámica } & $\begin{array}{l}\text { ¿Cuáles son las consecuencias del trabajo } \\
\text { infantil? }\end{array}$ & \\
\hline & $\begin{array}{l}\text { - Algunos Modelos Teóricos, incluyen un } \\
\text { análisis sobre la disponibilidad de crédito } \\
\text { como elemento de combate al trabajo } \\
\text { infantil. }\end{array}$ & $\begin{array}{l}\text { Jean Marie Baland y } \\
\text { James Robinson (1998) }\end{array}$ \\
\hline & $\begin{array}{l}\text { - Sobre los modelos con generaciones } \\
\text { solapadas. }\end{array}$ & $\begin{array}{l}\text { Gerhard Glomm } \\
\text { (1997); López-Calva } \\
\text { (2002) }\end{array}$ \\
\hline & $\begin{array}{l}\text { - Sobre las espirales virtuosas donde al } \\
\text { alcanzar un punto determinado de combatir el } \\
\text { trabajo infantil, se romperá el circulo vicioso } \\
\text { y éste se reducirá rápidamente. }\end{array}$ & D.P. Chaudhri (1997) \\
\hline & $\begin{array}{l}\text { - Sobre una discusión de las externalidades de } \\
\text { nivel educativo de los padres. }\end{array}$ & $\begin{array}{l}\text { Basu y James Foster } \\
(1998)\end{array}$ \\
\hline
\end{tabular}


RIS

REVISTA INTERNACIONAL DE SOCIOLOCI

№ 36, Septiembre-Diciembre, 2003

EDUARDO CALDERÓN CUEYAS

\section{El papel de la Economía del Bienestar o Normativa}

La economía del bienestar en materia de trabajo infantil pretende dar respuesta a tres casos interactivos: el primero relacionado con la búsqueda de la pura eficiencia de la política; el segundo en donde se consideran las implicaciones distributivas dentro del hogar y por último la distribución entre los hogares (Basu y Van, 1998).

Al buscar la eficiencia de la política, hace suyo el principio de externalidad que aplica la economía positiva y busca intervenir ante lo que considera como fallos de mercado. En el sector de la educación se presentan múltiples fallos como: si los rendimientos sociales de los estudios básicos son mayores que los rendimientos privados ${ }^{11}$ se genera la tensión entre la maximización de la función de utilidad social y las decisiones individuales, pues no sería ilógico pensar que un individuo racional preferiría por ello enviar a sus hijos al trabajo en vez de la escuela (Grootaert y Ravi, 1995). Frente a este punto ¿cuál debe ser la naturaleza de la intervención según la economía del bienestar?

Uno de sus postulados señala que ante fallos de mercado, la intervención más deseada (entiéndase eficiente) es a que se centra sólo en ese mercado. Por lo tanto, una política más integral que actúe en diferentes sectores sociales o bien que garantice los derechos de los niños no sería su prioridad. Su intervención solo la justificaría en buscar incrementar los rendimientos privados de la educación primaria para que se equipare al rendimiento social.

¿Pero qué pasaría si existe discriminación contra los niños aun cuando no existan fallos de mercado? ¿Qué diría la economía del bienestar? Este caso se presenta cuando dentro del hogar se le da menor valoración a la utilidad de los niños respecto a la de los propios padres o de la función de bienestar social. $\mathrm{La}$ economía de bienestar justifica su intervención estudiando el proceso de asignación dentro del hogar, es decir buscará encontrar los incentivos adecuados para que el jefe de familia que está decidiendo sobre el tiempo del niño para que trabaje cambie su decisión, pero sólo si esto implica poder alcanzar una escala mayor en la función de bienestar social, no para garantizar sus derechos. Sobre este punto y otros más se profundizará en el siguiente apartado.

\section{CRÍTICAA LA ECONOMÍA DE BIENESTAR}

Las politicas de intervención en materia de trabajo infantil necesariamente conllevan juicios valorativos, como ya se estudió en el apartado anterior y la rama de la Economía que se encarga de investigar al respecto es la Economía del Bienestar.

\footnotetext{
"Esto sucedería si se tiene el interés que mediante la educación básica se reduzca la tasa de fertilidad en donde en algunos países esto puede ser deseable desde el punto de vista social.
} 
Es importante primero tener claro dentro de la clasificación de teorías de la justicia dónde se ubica la Economía de Bienestar. Su formato es básicamente consecuencialista, es decir, determina un resultado al que debe llegar cualquier sociedad que quiera merecer la calificación de justa. En el lado opuesto estarían aquellas teorías llamadas deontológicas, que se limitan a establecer un conjunto de derechos y reconocen a una sociedad justa como aquélla que respeta estos derechos independientemente de las consecuencias.

A fin de entender mejor sus postulados y poder ir desarrollando una crítica a su base normativa con aplicación al estudio del trabajo infantil, es importante entender cómo ha sido su desarrollo desde el Utilitarismo Clásico hasta el Utilitarismo Ordinalista, o lo que algunos consideran como Nueva Economía de Bienestar (NEB). Para ello nos basaremos en el brillante estudio sobre ética y economía de Antoni Doménech (1996).

\section{Utilitarismo clásico y sus limitaciones con relación al Trabajo Infantil}

La primera economía de bienestar puede entenderse como una versión de la ética social utilitarista decimonónica clásica por lo menos hasta los años treinta del siglo XX (Doménech, 1996:192). En el Utilitarismo Clásico el distribuendum era la utilidad cardinal, entendida como el grado de satisfacción de los deseos o preferencias de los individuos ${ }^{12}$. La ventaja que vio el utilitarismo clásico en esta concepción de la utilidad eran sus propiedades métricas: poder asignarle un número cardinal y realizar operaciones como suma, resta, multiplicación, etc., y la comparabilidad interpersonal de utilidades.

Teniendo claro lo que se pretende distribuir, falta por identificar cuál es el criterio de distribución del utilitarismo clásico: es justa una sociedad que consigue maximizar la suma de las utilidades del conjunto de la sociedad, ahora bien, ¿cómo se puede interpretar esta teoría con relación al trabajo infantil?

Utilizando la ley de rendimientos decrecientes (LRD), uno de los supuestos más importantes del utilitarismo cardinalista, que dice que cuantos más recursos se tenga de un bien (consumo, actividades, etc,) una unidad adicional de ellos generará menos utilidad y a la inversa. Según lo anterior, para un gobierno cuyos principios sean utilitaristas su criterio de justicia podría ser aceptar una redistribución de los recursos expropiando a los ricos a favor de los pobres a fin de romper el círculo vicioso del trabajo infantil, pues por la LRD los pobres serían mayores generadores de utilidad porque parten de menos recursos. La redistribución llegaría hasta el punto en que el último céntimo arrebatado a los ricos generará en éstos

\footnotetext{
${ }^{12}$ Cabe señalar que aunque el distrubuendum sea la utilidad, en términos prácticos no se puede ir distribuyendo los deseos o preferencias entre los individuos; se tiene que hacer indirectamente, mediante recursos generadores de utilidad como el dinero, vales de despensa, entre otros mecanismos.
} 
RIS

REVISTA INTERNACIONAL DE SOCIOLOCIA

No 36, Septiembre-Diciembre, 2003

una des-utilidad igual al incremento de la utilidad del pobre, maximizándose la función de utilidad social agregada.

En teoría este argumento justificaría los programas orientados a combatir el trabajo infantil, ya que con su eliminación se estaría garantizando un incremento en las funciones de utilidad de los afectados (padres e hijos). El problema surge cuando se trata de responder hasta qué nivel debe llegar la redistribución y si se debería ponderar de distinta forma el incremento o disminución de las utilidades de los ricos y pobres tomando en cuenta elementos como las desigualdades de origen y desventajas físicas.

Aparte de esto, existen otros problemas de carácter ético, técnico-metodológicos y de criterio de distribución de la utilidad cardinal que la limitan como teoría de justicia en el análisis del trabajo infantil, como bien señala Doménech (1996):

1) Problemas éticos.

En su carácter de teoría consecuencialista, al utilitarismo clásico se le dificulta respetar los derechos de los individuos y los compromisos. Supongamos que dado un perfil de utilidades individuales en una sociedad, lo que maximiza la función de utilidad social sea una tasa de participación laboral de los niños del $20 \%{ }^{13}$. Debido a las condiciones estructurales y a las estrategias de crecimiento económico de una sociedad en particular, es muy posible que la maximización sólo se alcance mediante estas cuotas de trabajo infantil, incluso aceptando tipologías que conlleven condiciones de esclavitud como el realizado en la agricultura, el servicio doméstico, la industria del sexo, los sectores de confección de alfombras, producción de textiles, explotación de canteras y fabricación de ladrillos.

En estos casos, el utilitarismo estaría obligado a considerar como justo dicha tasa de participación de los niños o cualquier otra cifra que maximice la función agregada de utilidad sin respetar a priori los derechos individuales de los niños violando intuiciones morales básicas.

2) Problemas técnicos-metodológicos (distribuendum).

El utilitarismo clásico en su métrica de las funciones de utilidad individual no contempla un principio ético tan importante a la hora de realizar valoraciones normativas es el origen de los deseos y preferencias, como señala Rawls (1971). El debate surge al intentar definir si son legítimos o no las preferencias o deseos de un individuo, es decir, si nacen libres de cualquier tipo de coerción, influencia del entorno, o de restricciones económicas.

${ }^{13}$ Suponer esto no es descabellado ya que en las últimas estimaciones de la Organización Internacional del Trabajo la tasa de participación de los niños económicamente activos de 5 a 14 años por región son: África Sub-Sahariana 29 por ciento, Asia y el Pacífico 19 por ciento, Latinoamérica 16 por ciento, y el Medio Oriente junto con África del Norte del 15 por ciento (Every Child Counts ILO/ IPEC, 2002). 
En condiciones extremas como vivir en la pobreza, es posible que un padre se sienta satisfecho al enviar a su hijo a trabajar (efectos positivos en su función de utilidad), o que el mismo niño acepte su condición de trabajador y llegue a preferirla frente a otros tipos de actividad como asistir a la escuela, todo ello para reducir la disonancia cognitiva que provoca ese estado.

De presentarse este comportamiento estaría justificado una cuota de trabajo infantil (sea cual sea su tipo de actividad) porque al registrar valores positivos en sus utilidades, se estaría maximizando la función de utilidad social permitiéndose altos grados de desigualdad. Al parecer estarían entrando en juego mecanismos psicológicos adaptativos y culturales que influyen en las preferencias de los individuos con lo cual sus deseos no siempre pueden considerarse cómo legítimos. Vale la pena explorar un poco más cuáles son los efectos de las disonancia cognitiva sobre la teoría del utilitarismo cardinal. Los mecanismos psicológicos adaptativos como la disonancia cognitiva implican que la función de utilidad de un reductor de disonancias no será convexa, es decir, que en vez de aumentar la des-utilidad con un aumento de dosis de bienes desagradables como el trabajo infantil, la desutilidad tiende a disminuir, o de otra manera, aumenta su utilidad con lo cual en el efecto global se podría estar maximizando una función de utilidad social injusta según nuestras intuiciones morales (Akerloff y Dickens, 1982).

Otro punto que obvia en su formulación el utilitarismo clásico es la responsabilidad de los individuos sobre sus preferencias. Un proceso de distribución, en este caso de la utilidad cardinal, debe diferenciar entre los tipos de preferencias que estaría dispuesta a financiar la sociedad y en cuáles no debería hacerlo. Este tipo de distinción no la contempla el utilitarismo y permitiría situaciones donde se estén subvencionando deseos excéntricos como el consumo compulsivo de caviar de Agapito, del cual es éticamente responsable, y olvidar la satisfacción de la silla de ruedas de Torcuato (Doménech, 1996: 192).

Dentro del interés de este estudio, cabría preguntarse: ¿hasta qué punto los niños son responsables sobre sus preferencias, como por ejemplo la de trabajar? Aunque de aquí puede surgir un debate más extenso, me inclino a pensar que los niños no son aún individuos capaces de ejercer una decisión completamente libre y consciente, valorando sus consecuencias hacia su persona y hacia el bien común; y más aún si se encuentran inmersos en situaciones sociales desfavorables.

Lo anterior nos lleva a abordar el tema desde la responsabilidad de los padres hacia sus hijos, por lo tanto, ¿cuál debería de ser la responsabilidad de las preferencias de los padres hacia sus hijos? La intuición moral nos llevaría a concluir que el padre debe buscar el mayor bienestar de sus hijos, pero si nos encontramos ante una situación en que por sus condiciones de pobreza o bien por normas sociales, el padre adopta una actitud entre comillas irresponsable frente a sus hijos enviándolos a trabajar, ¿se podría justificar una intervención del Estado? ¿Debería existir algún tipo de penalización hacia los padres? El utilitarismo cardinal no contemplaría ningún tipo de intervención desde esta óptica. 
RIS

REVISTA INTERNACIONAL DE SOCIOLOGIA

№ 36, Septiembre-Diciembre, 2003

EDUARDO CALDERÓN CUEVAS

Finalmente, en este aparatado falta por mencionar un problema de gran influencia en el estudio del trabajo infantil, a saber, el conocido como el "dominio no restringido" o, en otras palabras el de la inclusión de preferencias inmorales en la función social agregada de utilidad. En la métrica de la utilidad cardinal se excluye toda información sobre los sentimientos interpersonales (amor, amistad, simpatía, altruismo, envidia, egoísmo), ello debido a que los primeros economistas de bienestar partieron de una teoría económica positiva fundada en el supuesto de que los agentes son racionales y maximizadores de funciones de utilidad independientes unas de otras y de cualquier tipo ${ }^{14}$. Estas funciones las consideran dadas en la sociedad y no las califican moralmente, con lo cual al distribuir los recursos ponderan por igual los deseos altruistas, generosos y solidarios que los deseos egoístas, envidiosos, sádicos e intolerantes.

El utilitarismo como teoría de justicia distributiva podría generar "dilemas éticos muy graves pues justificaría un proceso de redistribución hacia preferencias inmorales, ya que puede existir un individuo cuya preferencia sea demandar servicios de prostitución y pornografia infantil que le generaría altas utilidades, con lo cual estaría justificado su financiamiento.

Por otro lado, no se debe olvidar que una parte importante de las motivaciones para disminuir el trabajo infantil provienen de preferencias altruistas de los padres (al buscar invertir en el futuro de sus hijos), de los sentimientos solidarios (como los programas de apadrinamiento de los niños que viven en la calle, o trabajan en situaciones de riesgo), entre otras preferencias interpersonales. Lo anterior indica que una política distributiva cuyo objetivo sea el combate del trabajo infantil no puede dejar de calificar moralmente las preferencias y ponderar a la hora de distribuir los recursos con un mayor peso a aquéllas basadas en sentimientos altruistas o similares, siendo ésta una limitación del utilitarismo cardinal.

\section{3) Problemas de maximización de la suma de utilidades (criterio).}

Además de las dificultades antes señaladas, el utilitarismo presenta problemas con relación a su criterio de distribución. Pueden existir un conjunto de individuos que son malos generadores de utilidad o bienestar subjetivo, con lo cual el criterio de maximización podría excluirlos en su agregación de utilidades. En este sentido, se podría excluir a los niños trabajadores al buscar la maximización de la utilidad total por generar des-utilidades.

\footnotetext{
${ }^{14}$ En los estudios de la teoría formal de la utilidad y de elección social, la única manera y muy limitada en que se expresa el carácter altruista, envidioso, generoso, de las preferencias de los individuos consiste en hacer sus funciones de utilidad matemáticamente crecientes, indiferentes, o decrecientes respecto a la de los demás individuos (Doménech, 1996).
} 


\section{Del Utilitarismo a la Economía del Bienestar}

Las dificultades ya mencionadas, llevaron al grueso de la economía normativa a redefinir su base ética pero sin alejarse de los principios de la economía positiva. Ello derivó en lo que algunos han llamado la Nueva Economía de Bienestar, donde su distribuendum ya no sería la utilidad cardinal si no la ordinal ${ }^{15}$.

Esta variación ya no permite el uso como criterio de distribución de la maximización de la suma de utilidades debido a la imposibilidad de realizar operaciones aritméticas de las funciones de utilidad; por ello la NEB, inspirándose en la teoría económica, adopta un nuevo criterio normativo de justicia, el Óptimo de Pareto: la situación de mayor justicia "óptima" es aquella donde nadie puede mejorar su utilidad sin empeorar la del otro. Este criterio en principio parecería muy justo e indiscutible, pero ¿es suficiente como criterio de justicia distributiva? ¿qué dificultades plantea?

Una teoría normativa que se conforme con este criterio sería muy poco informativa (Doménech, 1996). Tendría serios problemas metodológicos y éticos, ya que el óptimo de Pareto es compatible con estructuras socio-económicas muy dispares y desiguales; de hecho este principio se desarrolló para evitar la necesidad de emitir juicios sobre un proceso distributivo, como afirma Sen (1970).

Si sólo nos limitáramos a afirmar que una sociedad justa es aquella económicamente eficiente, cualquier intento de redistribución en pequeña o gran escala para disminuir una situación inicial altamente desigual, o bien para aplicar una política de redistribución inter-clase social que elimine situaciones como el trabajo infantil, no sería compatible con la NEB, ya que en este intento se estaría perjudicando la utilidad de unos para mejorar la de otros.

Ante esta dificultad, la NEB introdujo un mecanismo complementario que permitiera seleccionar entre distintos óptimos de Pareto, la elección social, y así recuperar de nuevo su capacidad selectiva e informativa que había perdido por el cambio ordinalista. Sin embargo, en 1951 Arrow demostró con un teorema matemático la imposibilidad de una combinación paretiana con cualquier mecanismo de elección social ${ }^{16}$.

\footnotetext{
${ }^{15}$ Medir ordinalmente algo significa conformarse únicamente con la información acerca del orden de las preferencias de los individuos renunciando a su intensidad y a la comparabilidad interpersonal.

${ }^{16} \mathrm{El}$ teorema consiste en demostrar que no se puede respetar simultáneamente un conjunto de seis condiciones básicas que debe cumplir un mecanismo de elección social cuando hay al menos tres alternativ disponibles. Las seis condiciones son: a) Dominio no restringido de la función de elección socıal, es decir, que se garantice que todas la ordenaciones individuales de preferencias serán tomadas en cuenta por la función de elección social; b) Exogeneidad y estabilidad de las preferencias en los procesos de elección social; c) Racionalidad Colectiva, es decir, que la función de elección social garantice alguna condición débil de transitividad; d) Independencia de las alternativas irrelevantes; e) Optimalidad Paretiana; f) No Dictadura.
} 
R IS

REVISTA INTERNACIONAL DE SOCIOLOCIA

$N^{\circ} 36$, Septiembre-Diciembre, 2003

EDUARDO CALDERÓN CUEVAS

Frente a este cuestionamiento en la base de la teoría de la NEB se puso en evidencia la necesidad de buscar criterios alternativos para definir una sociedad justa en materia distributiva. En el siguiente apartado se reflexiona sobre otros criterios de justicia cuyas implicaciones en materia de trabajo infantil vale la pena destacar, como la propuesta del principio de diferencia de Rawls, la teoría de igualdad de oportunidades de Roemer, y las capacidades y funcionamientos de Amartya Sen.

\section{CRITERIOS ALTERNATIVOS DE JUSTICIA DISTRIBUTIVA}

La aparición en 1971 de la Teoría de la Justicia de Rawls, libre de muchas de las limitaciones de la economía del bienestar, abre el campo a nuevos conceptos de justicia y su aplicación distributiva. Propone un distribuendum totalmente distinto a la utilidad que son los bienes primarios ${ }^{17}$ y su criterio de distribución es el leximin.

Su criterio está basado en respetar el siguiente orden de prioridades lexicográficas: 1) una sociedad deberá organizar un sistema de libertades públicas igual para todos, 2) deberá garantizar la igualdad equitativa de oportunidades de acceso a cargos y posiciones, es decir, no se puede discriminar a nadie por características étnicas, raciales, de género, entre otras, y 3 ) asegurado lo anterior, se debe distribuir la riqueza de modo que se maximicen los ingresos de los más desfavorecidos principio de diferencia.

Asimismo, las dos grandes implicaciones del abandono del utilitarismo son: a) hacer a los individuos responsables de sus propias preferencias; y b) se concibe a los individuos como personas distintas y separadas.

Lo anterior implicaría que las desigualdades existentes en una sociedad que no son producto de las responsabilidades de los individuos, deben compensarse de alguna forma (contrarrestar los efectos del azar social). ${ }^{18}$ Por tanto, desde esta teoría estaría completamente justificada una intervención del Estado para garantizar que las preferencias de los padres sean completamente ajenas a cualquier tipo de restricción, principalmente la económica.

\footnotetext{
${ }^{17}$ Los bienes a los que hace referencia son: libertades fundamentales, como el derecho al voto, de expresión, conciencia y pensamiento, de propiedad personal, oportunidades de acceso a las posiciones sociales y ventajas socioeconómicas, como la renta y riqueza. También contempla bienes naturales como salud y talentos.

${ }^{18}$ Es importante destacar que la teoría rawlsiana no es sensible al azar natural.
} 
Rawls estaría a favor de políticas con tratamiento diferenciado hacia los niños para que no se vea obstaculizado su desarrollo físico y mental. Apoyaría la idea de la irresponsabilidad de los niños ante sus preferencias; por lo tanto, si los padres no pueden garantizarle un futuro con igualdad de oportunidades de acceso a distintas posiciones sociales, una educación continua, un determinado nivel de ocio, entre otros de los bienes primarios a los que hace referencia, el gobierno debería adoptar una actitud "paternalista" y compensar al niño mediante programas integrales de protección, desarrollo y elevación de la renta de los peor situados ${ }^{19}$.

Sin embargo, la crítica de Rawls al utilitarismo sobre la irresponsabilidad de los individuos sobre sus preferencias es muy potente en cuestionar la igualación del bienestar (utilidades) más no justifica la métrica de los bienes primarios. Cohen (1993) argumenta de forma precisa que en la medida en que las personas son realmente responsables de sus gustos, los déficit significativos de bienestar no requieren la atención de la justicia. Por lo tanto, se debe compensar sólo aquellos déficit de bienestar que no puedan atribuirse correctamente a elecciones individuales. Se debe reemplazar la igualdad de bienestar por igualdad de oportunidades para tener el bienestar (Cohen, 1993: 32).

Este planteamiento nos lleva a discutir sobre la teoría de igualdad de oportunidades y su relación con el TI. Roemer (1998) aborda de forma muy precisa esta teoría y señala que existen dos formas de entender el concepto de igualdad de oportunidades: la primera se refiere al principio no-discriminatorio basado en que los individuos deben ser juzgados por su desempeño en las labores que se les encomienda y no por ciertos atributos como raza, sexo, nivel económico, religión, entre otros. La segunda, como él lo explica, se basa en la idea de "nivelar el campo de juego de los competidores", especialmente en la etapa formativa de las personas.

La esencia del concepto de igualdad de oportunidades y la clave entre estos dos enfoques es determinar hasta qué grado es responsable el individuo del logro de objetivos particulares como educación, empleo, salud y nivel de ingresos. Roemer establece la diferencia temporal entre un "antes" y "después" en la noción de igualdad de oportunidades: antes de la competición por una ocupación social se deben igualar las condiciones; pero después de que ésta empiece, los individuos van por la libre. ¿Cómo definimos ese punto de partida de competición? ¿Cómo se puede diferenciar entre aquellos individuos a los que se debe compensar con una política de igualdad de oportunidades y a los que no? ¿Qué se puede decir sobre el trabajo infantil?.

\footnotetext{
${ }^{19}$ En este sentido sólo se podrían considerar como justas las desigualdades que, por contribuir a la eficiencia, se traducen en una elevación del nivel de renta de los peor situados.
} 
R I S

REVISTA INTERNACIONAL DE SOCIOLOCIA

№ 36, Septiembre-Diciembre, 2003

EDUARDO CALDERÓN CUEVAS

Roemer propone un algoritmo que permita reflejar el concepto de igualdad de oportunidades acorde con cualquier espectro de ideas sobre la responsabilidad del individuo y poder delimitar el punto de partida de competición ${ }^{20}$.

Asimismo, para poder diferenciar a los individuos que deben recibir los beneficios de una política de igualdad de oportunidades, Roemer introduce el concepto de control sobre las circunstancias. Señala que debe diferenciarse entre los tipos de circunstancias que están fuera del control del individuo (genes, cultura, antecedentes familiares), y sus niveles de esfuerzo y voluntad propia. Esto indicaría que las diferencias, por ejemplo en el desempeño académico de un niño, no deben compensarse si provienen de diferencias de esfuerzo o de la voluntad propia del niño (asistir o no a clase).

Por tanto, ¿debería existir alguna diferenciación de esfuerzos?, ¿qué diría Roemer sobre compensar el esfuerzo extra que realiza un niño que trabaja y estudia por su condición de pobreza, frente a uno que sólo trabaja? Roemer estaría a favor de una diferenciación de esfuerzos pues entiende que éstos en determinado nivel están condicionados por las circunstancias. Reconoce mediante su ejemplo de Alan y Betsy ${ }^{21}$ que existe una gran gama de tipos de distribuciones de esfuerzo que puede presentar un niño en función de su condición de pobreza, de si trabaja o no, de sus orígenes sociales.

Frente a esto, determina que el criterio de distribución que debe seguir una política de igualdad de oportunidades será el de buscar igualar los resultados de todos aquellos individuos que se encuentren en el mismo percentil de su tipo de distribución de esfuerzos.

Con el criterio de Roemer se puede avanzar aún más en relación al TI; aparte de sus reflexiones sobre los esfuerzos, ¿qué se puede decir sobre la responsabilidad de los niños frente a sus preferencias? Roemer, establece una diferenciación entre la responsabilidad moral, a lo que llama "responsable", y el ser responsable frente a los actos o accountable. Considera que hacer a un individuo "responsable" no significa que siempre lo hagamos accountable, es decir, un individuo puede ser moralmente responsable de su comportamiento aunque esté influido por sus cir(1998).

${ }^{20}$ Los interesados en ver su desarrollo formal pueden consultar el Capitulo 4 de Roemer

${ }^{21}$ Aplicando su ejemplo al trabajo infantil sería lo siguiente: Alan es un niño que combina el trabajo con el estudio (tipo 1) y Betsy sólo estudia (tipo 2). Alan ha realizado un esfuerzo de nivel 5 que lo ubica en el percentil noventa de la distribución de esfuerzos de este tipo de niños, y Betsy ha realizado un esfuerzo también del nivel 5 pero se encuentra en la media de la distribución de esfuerzos del tipo 2. Estadísticamente se podría inferir que de un grupo amplio de niños que se criaran bajo las mismas circunstancias del tipo 1, tan sólo un $10 \%$ realizaria un mayor esfuerzo que Alan, a diferencia de si se criaran bajo las condiciones del tipo 2, en las que un $50 \%$ de niños realizarían un mayor esfuerzo que Betsy. Lo anterior indica la necesidad de compensar de distinta forma el esfuerzo realizado por Alan. 
cunstancias, pero sobre la responsabilidad de sus actos y consecuencias se pueden realizar excepciones como en el caso de niños en que las circunstancias de crianza y de dependencia de sus padres pueden justificar las políticas de compensación. Entonces, según la teoría de igualdad de oportunidades, un niño sólo puede ser responsable de sus grados de esfuerzo pero no de sus niveles determinados por sus circunstancias ${ }^{22}$.

Una teoría de justicia distributiva que iría mas allá de las propuestas de Rawls y Roemer con relación al Trabajo Infantil es la de capacidades de Amartya Sen. Aceptando el criterio de leximin de distribución, Sen propone una nueva teoría de la justicia con un cambio de métrica. A Sen le preocupan las diferencias entre los individuos en cuanto a sus capacidades para convertir los recursos en libertades reales. Es decir, las capacidades de Sen representan libertades reales, mientras que los bienes primarios rawlsianos representan sólo los medios para la libertad (Doménech, 1996).

Sen crítica la teoría de Rawls sobre la propuesta de los bienes primarios, argumentando de forma sencilla pero profunda sobre la irrelevancia de preocuparse por los bienes como tales, excluyendo el juicio sobre lo que los bienes hacen por el ser humano. Sen distingue el concepto de capacidad con el de funcionamientos que representan las cosas que se logran hacer o ser al vivir. La capacidad, por tanto, sería una combinación de alternativas de los funcionamientos que el individuo puede lograr, entre los cuales puede elegir una colección (Sen, 1993).

De esta forma, Sen estaría a favor de una intervención del Estado para igualar las capacidades de un individuo a fin de que pueda elegir entre estilos de vida alternativos. Por tanto, no dudaría en aplicar políticas distributivas hasta evitar por completo el trabajo infantil para garantizar el pleno desarrollo de las habilidades y conocimientos de los niños como condición básica de igualación de funcionamientos. En este sentido, podría decirse que su teoría es un híbrido entre consecuencialista y deontológica, porque defiende al mismo tiempo el derecho a la libertad real de elegir un determinado tipo de vida sin condiciones, y pretende una igualación de funcionamientos para garantizar un resultado justo.

Por tanto, bajo la teoría de Sen, se pueden apoyar políticas categóricas destinadas a ampliar las capacidades de los niños que trabajan, tales como: establecer centros de enseñanza en su lugar de trabajo; proporcionar servicios de apoyo a los padres, en particular a las madres; y suministrar servicios a los niños que trabajan en la calle (planes de alimentación, albergues nocturnos, programas de alfabetización), entre otros.

\footnotetext{
${ }^{22}$ En el ejemplo anterior, se puede decir que Betsy deberá asumir una mayor responsabilidad de sus actos (es más accountable) por su menor grado de esfuerzo educacional que Alan y, por tanto, una política de igualdad de oportunidades deberá compensarlo de alguna forma.
} 
RIS

REVISTA INTERNACIONAL DE SOCIOLOCIA

№ 36, Septiembre-Diciembre, 2003

EDUARDO CALDERÓN CUEVAS

También entrarían en este conjunto políticas de carácter legislativo que busquen garantizar los derechos de los niños, tales como una aplicación más estricta de la ley, como por ejemplo contra aquellos que se dedican al tráfico de niños; y más aún, su esquema de justicia deja la puerta abierta a políticas que modifiquen los valores culturales y normas sociales que toleran la explotación económica de los niños.

\section{CONCLUSIONES}

La discusión presentada en este documento señala que el estudio del Trabajo Infantil conlleva una gran carga y.variedad de juicios normativos para definir los tipos de políticas distributivas y su alcance. A través del análisis de los principales postulados de la Economía de Bienestar (tanto del utilitarismo clásico como de la Nueva Economía de Bienestar) se puede concluir que como teoría de la justicia distributiva presenta importantes limitaciones para integrar en su marco conceptual situaciones, como las del trabajo infantil, y poder justificar políticas distributivas más profundas, como las que se requieren a fin de eliminar esta problemática social que caracteriza al subdesarrollo y a la pobreza.

Sobre la naturaleza de las políticas dirigidas a combatir el trabajo infantil, se debe tener clara la necesidad de separarlas en dos enfoques, como señala Anker (2000): la primera, centrada en eliminar las peores formas de trabajo infantil (como las que se dan en actividades ilícitas, en la prostitución, el tráfico infantil, la guerra, entre otras) mediante legislaciones y políticas de control. La segunda, deberá actuar sobre los tipos de trabajo infantil inaceptables que perjudican el desarrollo físico, mental y psicológico del niño, apoyándose en programas que mejoren la calidad de las escuelas, así como en políticas macro que garanticen a las familias de escasos recursos alternativas de ingreso para que no utilicen al niño como seguro de desempleo.

Las limitaciones más relevantes del utilitarismo clásico en el análisis del trabajo infantil son producto de su distribuendum (utilidades) y de su criterio de distribución entendido como la maximización de la suma de utilidades del conjunto de la sociedad y se pueden clasificar en tres:

1) Los problemas éticos que se derivan de su carácter de teoría consecuencialista. El proceso de distribución no garantiza el respeto de los derechos de los niños sino sólo mira por las consecuencias del mismo; por tanto, se pueden llegar a resultados intuitivamente inaceptables, como justificar una cuota de trabajo infantil que maximice la función de utilidad agregada.

2) Los problemas técnicos metodológicos implícitos al tomar como distribuendum las utilidades. Este apartado se puede subdividir a su vez en tres puntos y cuya relevancia normativa con relación al trabajo infantil es vital: 
- Sobre el origen de los deseos y preferencias: ¿Qué tan libres están las preferencias de los individuos (padres e hijos) de cualquier influencia del entorno? ¿Se debe compensar a quien pueda registrar altos niveles de utilidad como efecto de la reducción de la disonancia cognitiva dada entre el estado de pobreza o trabajo infantil? Ni el utilitarismo ni la NEB consideran este tipo de preguntas para justificar algún tipo de política compensatoria.

- Sobre la responsabilidad de los individuos sobre sus preferencias:¿cuál debería ser la responsabilidad de las preferencias de los padres hacia sus hijos? ¿Se debería justificar la intervención del Estado ante decisiones irresponsables de los padres? Dado que el utilitarismo y la NEB omiten cualquier valoración al respecto, se presenta un vacío muy importante como teoría de justicia distributiva.

- Sobre el problema del dominio no restringido o de preferencias inmorales: el utilitarismo y la NEB generan dilemas éticos muy graves, ya que en su esquema teórico justificarían un proceso de distribución hacia preferencias inmorales si éstas generaran altos niveles de utilidad, como en el caso de algún individuo cuya preferencia y felicidad consistiera en demandar servicios de prostitución y de pornografia infantil.

3) El problema de agregación de las utilidades de los individuos que son malos generadores de utilidad o bienestar subjetivo, como aquellos que tienen disminuciones físicas o psíquicas, por vivir en la pobreza, o por tener que trabajar en vez de estudiar. En la agregación se podría excluir a este tipo de individuos cuando el objetivo es maximizar la utilidad total, ya que estos generan des-utilidades.

El utilitarismo sobrevivió a su duro escrutinio durante mucho tiempo debido a la ausencia de una teoría de la justicia tan informativa como ésta. Posteriormente, la Nueva Economía de Bienestar al intentar superar las deficiencias del utilitarismo con el abandono de la métrica cardinal inter-personalmente comparable a una reformulación en clave ordinalista, aplicando el criterio del óptimo de Pareto, no hizo más que abrir nuevos retos a superar tras la demostración del teorema de imposibilidad de Arrow.

Un camino alternativo se presentó con el desarrollo de nuevas teorías de justicia distributiva como las de Rawls, Roemer y Sen, cuya aportación es importantísima a la valoración y clasificación de lo que debe ser una sociedad justa, así como a los tipos de políticas distributivas que son necesarias para llega a este fin. Mediante la reflexión sobre los criterios de justicia como el principio de diferencia, las capacidades y funcionamientos y la igualdad de oportunidades, se fabrican nuevas llaves para intentar abrir la gran puerta que conduce a una sociedad justa en donde no exista el trabajo infantil. 


\section{REFERENCIAS BIBLIOGRÁFICAS}

AKERLOFF, G. y W. DICKENS (1982), "The Economic Consequences of Cognitive Disonante", American Economic Review, vol. 72, n. ${ }^{\circ}$, pp. 307-319.

ANKER, R. (2000), “The Economics of Child Labor: A framework for Measurement”, International Labor Review, vol. 139, n 3 .

BASU, A. (1993), "Family Size and Child Welfare in a Urban Slum: Some Disadvantages of Being Poor but Modern", en Lloyd C. B (coord.), Fertility, Family Size and Structure: Consequences for family and children, New York, Porceedings of a Population Council Seminar, 9-10 June 1992, The Population Council.

BASU, K. (1999), "Child Labor: Cause, Consequence, and Cure with Remarks on International Labor Standars”, Jounal of Economic Literature, vol. XXXVIII, pp. 1083-1119.

BASU, K. y H.V. PHAM (1998), "The Economics of Child Labor", American Economic Review, vol. 88, n. $^{\circ} 3$, pp. $412-427$.

BONNET, M. (1993), "Child Labor in Africa”, International Labor Review, vol. 132, n. ${ }^{\circ} 3$, pp. 371-389.

COHEN, G.A. (1993), “Igualdad de qué? Sobre el Bienestar, los Bienes y las Capacidades” en Nussbaum, C. M. y Sen A. (coord.), La Calidad de Vida, México, Fondo de Cultura Económica, 1996, pp. $27-53$

CUNNINGHAM, H. (1990), "The Employment and Unemployment of Children in England 16801851 ", Past and Present, n' 126, pp.115-50.

DEGRAFF, D.S. et al. (1993), "The Implications of High Fertility for Children Time Use in Philippines", en Lloyd C. B (coord.), Fertility, Family Size and Structure: Consequences for family and children, New York, Porceedings of a Population Council Seminar, 9-10 June 1992, The Population Council.

DOMÉNECH, A. (1996), "Ética y Economía de Bienestar: Una Panorámica”, en Gvouciglia Osvaldo, Cuestiones Morales, Madrid, Trotla, pp.191-222.

GROOTAERT, C. (1998), "Child Labor in Cote d'Ivoire: Incidence and Determinants", The Policiy Analysis of Child Labor: A Comparative Study, Christiaan Grootaert and Harry Patrinos (editores), World Bank, Washington D.C.

GROOTAERT, C. y R. KANBUR (1995), “Child Labour: An Economic Perspective”, International Labour Review, vol. 134, n. ${ }^{\circ}$, pp. 187-203.

GUPTA, M.R. (1997), "Unemployment of Adult Labour and the Supply of Child Labour: A Theoretical Analysis", Mimeo, Jadaupur U. Calcutta.

GUSTAFSSON-WRIGHT y HNIN H. PYNE. (2002), "Gender Dimensions of Child Labor and Street Children in Brazil", World Bank Research, working paper 2897, October. 
JACOBY, H. y E. SKOUFIAS (1994), "Risk, Financial Markets and Human Capital in a Developing Country”, Mimeo, World Bank Policy Research Department, Washington, D.C. World Bank.

INTERNATIONAL LABOR OFFICE (2002), "Every Child Counts", Child Labor, IPEC, http// www.ilo.org.

LEVISON, D., S.K. MOE y F.M. KNAUL (2001), "Youth Education and Work in Mexico", World Development, vol. 29, n. ${ }^{\circ} 1$, pp. 167-188.

LLOYD, C.B. (1993), "Fertility, Family Size and Structure- Consequences for Family and Children", Porceedings of a Population Council Seminar, Nueva York, 9-10 June 1992, Nueva York, The Population Council.

LÓPEZ-CALVA, L.F. y A. RIVAS (2002), “Acumulación de Capital, Trabajo Infantil y Educación”, El Trimestre Económico, México. vol. LXIX, n. ${ }^{\circ} 273$. pp. 3-36.

LÓPEZ-CALVA, L.F. (2001), "Child Labor: Myths, Theories and Facts", Journal of International Affairs, Nueva York, vol. 5, n. ${ }^{\circ}$ 1. pp. 59-73.

PSACHAROPUOLOS, G. (1997), "Child Labor Versus Educational Attainment: Some Evidencie from Latin America", J. Population Econ., 10: 4, pp. 337-86.

ROEMER, J. (1996), Theories of Distributive Justice, Cambridge, Harvard University Press.

SEN, A.K. (1993), "Capacidad y Bienestar", en C.M. Nussbaum y A. Sen (coord.), La Calidad de Vida, México, Fondo de Cultura Económica, 1996, pp. 54-83.

UNICEF (1993), Street and Working Children, Innocenti Global Seminar, Summary Report. 